\title{
PREVALENCE AND CORRELATION OF NASAL SEPTUM DEVIATION, CONCHA BULLOSA AND MAXILLARY SINUSITIS IN A GROUP OF ADULT EGYPTIAN POPULATION: A RETROSPECTIVE CBCT STUDY
}

\author{
Dina F. Ahmed*
}

\begin{abstract}
Objectives: The aim of this study is to determine the prevalence of NSD, CB and maxillary sinusitis and to investigate their possible correlation using CBCT in a group of adult Egyptian population.

Subjects \&methods: In this retrospective study, 75 CBCT scans of both sexes (29 males \& 46 females) in the age range between 13-61 years were selected from the archives of the Oral and Maxillofacial Radiology Department, Faculty of Dentistry, Cairo University. The presence of nasal septal deviation (NSD), concha bullosa (CB) as well as maxillary sinusitis and their correlation was assessed by an experienced Oral and Maxillofacial Radiologist.

Results\& conclusions: The results of this study showed that maxillary sinusitis is quite common $(62.7 \%)$ in the studied population. The prevalence of maxillary sinusitis was more common than both NSD and CB. No correlation between all examined parameters was found except an association between NSD and contralateral CB. There was also no statistically significant difference between both genders in all parameters. From the results of this study it can also be concluded that neither NSD, nor CB increases the incidence of maxillary sinusitis.
\end{abstract}

KEYWORDS: Nasal septal deviation, Concha bullosa, Maxillary sinusitis, CBCT

\section{INTRODUCTION}

The nasal septum is an important support and physiological structure of the nose ${ }^{1}$. It is located in the midline of the nasal cavity and divides the nose to form the two nostrils. The nasal septum contains both bone and hyaline cartilage and its thickness is about $2 \mathrm{~mm}^{2,3}$. Nasal septal deviation (NSD) is a malalignment of the nasal septum from the midline. NSD occurs only in humans and is the most common deformity of the nose. It is also considered as the most common anatomic variation ${ }^{4}$. Earlier studies stated that some sort of deviation was seen in almost $80 \%$ of healthy adults ${ }^{5,6}$. According to the literature, NSD occurs secondary to trauma during intrauterine life, birth, or any time during life ${ }^{7}$. It is reported that

* Lecturer of Oral and Maxillofacial Radiology, Faculty of Dentistry, Cairo University 
NSD may be related to sleep apnea, nosebleeds, repetitive sneezing, difficulty in breathing, and chronic sinusitis ${ }^{8}$. The deviation of the nasal septum causes change in the air flow in the nasal cavity, nasal cycle and mucociliary clearance. Although not always symptomatic, NSD frequently leads to respiratory problems due to the reduction of volume in the nasal cavity. Hypertrophy of the turbinates on the contralateral side of the septal convexity is also often noticed ${ }^{1,2,3,7}$.

Other studies investigated the relation between variants in the sinonasal anatomy and the occurrence of sinus pathology ${ }^{\mathbf{9}, 10,11}$. Concha Bullosa (CB) also known as middle turbinate pneumatization or aerated turbinate, is a common anatomic variant in the nasal cavity and may occur unilaterally or bilaterally ${ }^{12}$. The correlation between the presence of $\mathrm{CB}$ and contralateral NSD has been reported in different studies 12,13. However, the claim that presence of NSD and pneumatization of the conchae are potential contributors to the development of sinus disease is still a matter of debate ${ }^{14}$. While some believe that NSD or the presence of CB affect proper nasal airflow and cause sinonasal obstruction, and hence predispose to sinus disease ${ }^{\mathbf{1 5}, 16}$, others showed inconsistent findings ${ }^{17,18}$.

During the past years, CBCT became an important radiographic modality in numerous dental applications as it offers 3-dimensional images at a lower radiation dose than multislice CT (MSCT). However, for otolaryngologists, the potential use of CBCT in their field is not yet fully explored. Anatomical variations as well as pathological conditions within the nasal cavity and the surrounding paranasal sinuses are clearly demonstrated on CBCT images and are often reported as incidental findings in CBCT reports. With its high spatial resolution exceeding that of $\mathrm{CT}$ and its lower radiation dose, cone-beam imaging will definitely become the reference radiographic method in sinus pathology evaluation in the upcoming years ${ }^{19}$.
Although numerous studies investigated the relation between NSD and $\mathrm{CB}$ on maxillary sinusitis ${ }^{\mathbf{1 4 , 9}}$, only few of them studied such a correlation using CBCT ${ }^{10}$. Therefore, the intention of this study is to determine the prevalence of NSD, $\mathrm{CB}$ and maxillary sinusitis in a group of adult Egyptian population and to investigate their possible correlation using $\mathrm{CBCT}$.

\section{SUBJECTS AND METHODS}

For this retrospective study, 75 CBCT scans of both sexes ( 29 males $\& 46$ females) with a mean age of $35.44 \pm 13.48$ years and an age range between 13-61 years were selected from the archives of the Oral and Maxillofacial Radiology Department, Faculty of Dentistry, Cairo University. All selected scans were for patients referred for a CBCT examination for a different diagnostic purpose other than the purpose of this study; mostly for implant or orthodontic planning. As all the scans were obtained from a dental hospital, no information about the otolaryngological condition of the subjects was available. All CBCT scans were performed with the Planmeca ProMax 3D Mid machine (Helsinky, Finland). For standardization, all selected scans, were taken with the same parameters: $90 \mathrm{kVp}, 8 \mathrm{~m} \mathrm{~A}$ and $0.4 \mathrm{~mm}$ voxel size and 100x200mm field of view and showing the entire nasal cavity and maxillary sinus. Images were processed and analyzed with the Romexis Viewer 4.5.0.R on a personal computer running Microsoft Windows 10 (Microsoft Corp, Redmond, WA, USA).

This study excluded patients with history of malignant lesions, traumatic injuries and/or surgery in the sinonasal or maxillofacial region. Moreover, patients with clinical and/or radiographic manifestations of developmental anomalies, head and neck syndromes and those with systemic diseases affecting growth and development were excluded from the study.

The presence of NSD, CB, as well as maxillary sinusitis was assessed by an experienced Oral and 
Maxillofacial Radiologist. NSD was defined as right or left deviation of greater than $4 \mathrm{~mm}$ from the midline according to Smith et al ${ }^{\mathbf{1 0}}$. Presence of $\mathrm{CB}$ was considered when there was pneumatization of any size in the middle turbinate ${ }^{20}$. According to Rak et al. ${ }^{21}$, presence of maxillary sinusitis was considered when there was $3 \mathrm{~mm}$ or more mucosal thickening.

To evaluate NSD, an axial cut of $0.4 \mathrm{~mm}$ thickness that shows the anterior nasal spine was selected. On that axial cut, the line corresponding to the sagittal cut was adjusted to pass through the middle of the anterior nasal spine. Next, a coronal cut that shows both the anterior nasal spine and crista galli was selected to confirm that the midline passes through them both. The presence or absence of NSD was determined from that same coronal cut. In case of presence of deviation $(>4 \mathrm{~mm}$ from the midline), the direction of deviation whether right or left was assessed. The direction of deviation was determined by the side of the convexity of the nasal septum.

For determining the presence of $\mathrm{CB}$, several coronal cuts were evaluated. $\mathrm{CB}$ was then classified as (right, left or bilateral).

Presence of maxillary sinusitis was also assessed from $\mathrm{CBCT}$ coronal cuts. In case of presence of

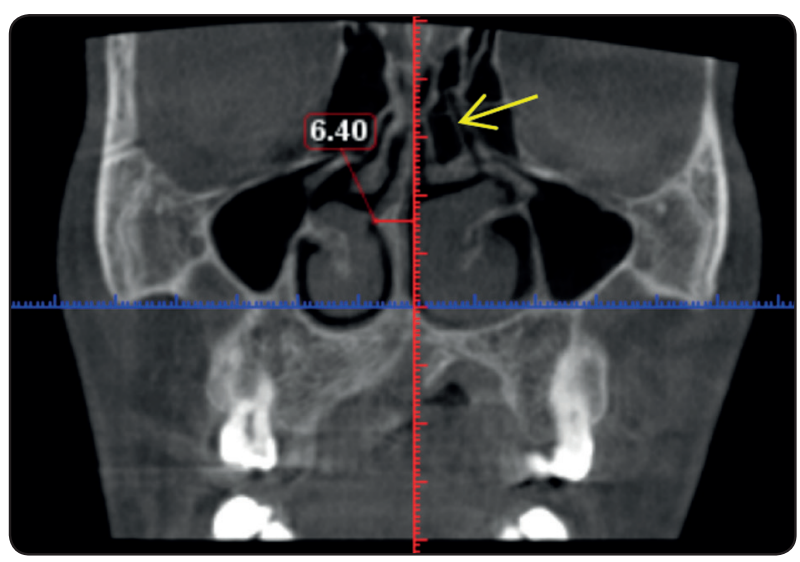

Fig. (1): Coronal CBCT scan showing right-sided NSD, measurement of its maximum extension from the midline and contralateral $\mathrm{CB}$

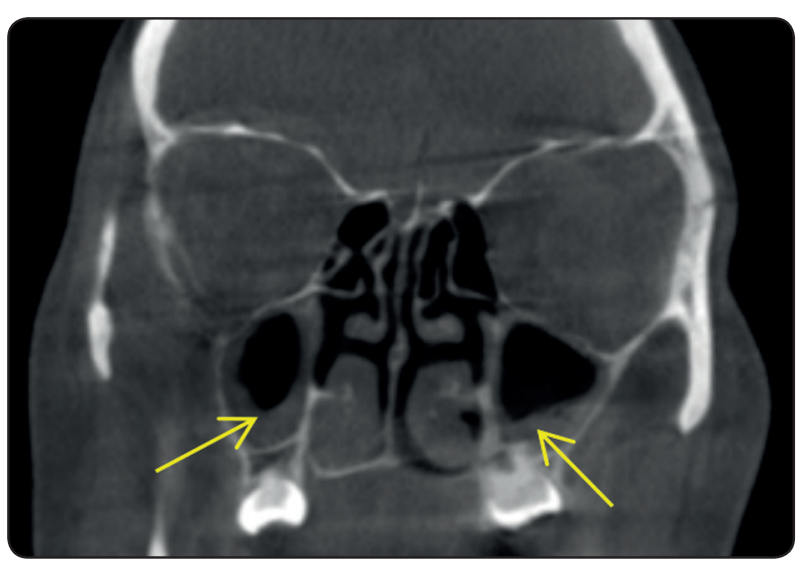

Fig. (2): Coronal CBCT scan showing bilateral moderate maxillary sinusitis

sinusitis, the side of sinusitis was described as right, left or bilateral. To determine the severity of maxillary sinusitis, the following scoring system was used for each side separately (mild: $<1 / 3$, moderate: $1 / 3-2 / 3$, severe: $>2 / 3$ of the maxillary sinus).

\section{Statistical analysis}

Data were collected, revised, coded and entered to the Statistical Package for Social Science (IBM SPSS) version 23. The distribution of quantitative data was tested by Kolmogorov-Smirnov test of normality. The quantitative data were presented as mean, standard deviations and ranges according to parametric distribution. Also qualitative variables were presented as number and percentages. The comparison between groups regarding qualitative data was done by using Chi-square test and/ or Fisher exact test when the expected count in any cell was found less than 5. The comparison between two independent groups with quantitative data and parametric distribution was done by using Independent t-test. The confidence interval was set to $95 \%$ and the margin of error accepted was set to $5 \%$. So, the p-value was considered significant at the level of $<0.05$. 


\section{RESULTS}

\section{Prevalence of NSD}

In the current study, NSD was seen in $28(37.3 \%)$ patients. $10(35.7 \%)$ of them were males and 18 $(64.3 \%)$ were females. There was no statistically significant difference between males and females regarding the presence of NSD $(\mathrm{p}=0.685)$. The mean age of patients with NSD was $37.79 \pm 12.99$ years and their age range was from 16-61years.

Right-sided NSD was seen in16 (57.1\%) of the patients while left-sided NSD was observed in 12 $(42.9 \%)$ of the patients. The mean of the maximum extension of NSD from the midline was $6.36 \pm 1.63$ $\mathrm{mm}$ and the range was from 4-10.03 $\mathrm{mm}$ (Table 1).

\section{Prevalence of $\mathrm{CB}$}

$30(40.0 \%)$ of the scans reviewed showed presence of CB. $4(13.3 \%)$ were located in the right side, $11(36.7 \%)$ were located in the left side and $15(50.0 \%)$ were present bilaterally. $13(43.3 \%)$ of them were males and17 (56.7\%) were females. Although females showed higher prevalence of $\mathrm{CB}$, there was no statistically significant difference between them $(\mathrm{p}=0.498)$.

The mean age of patients with $\mathrm{CB}$ was $30.53 \pm$ 12.26 years and the age range was from13-58years (Table 1).

TABLE (1): Prevalence, gender distribution of NSD, CB and maxillary sinusitis

\begin{tabular}{|c|c|c|c|c|}
\hline \multicolumn{3}{|c|}{ Presence of NSD (28) } & \multirow[t]{3}{*}{ p-value } & \multirow[t]{3}{*}{ Significance } \\
\hline \multirow{2}{*}{ Age } & Mean \pm SD & $37.79 \pm 12.99$ & & \\
\hline & Range & $16-61$ & & \\
\hline \multirow{2}{*}{ Sex } & Females & $18(64.3 \%)$ & \multirow{2}{*}{0.685} & \multirow{2}{*}{ NS } \\
\hline & Males & $10(35.7 \%)$ & & \\
\hline \multirow{2}{*}{ Direction of NSD } & Right & $16(57.1 \%)$ & & \\
\hline & Left & $12(42.9 \%)$ & & \\
\hline \multirow{2}{*}{ Maximum extension of deviation } & Mean \pm SD & $6.36 \pm 1.63$ & & \\
\hline & Range & $4-10.03$ & & \\
\hline \multicolumn{3}{|c|}{ Presence of CB (30) } & p-value & Significance \\
\hline \multirow{2}{*}{ Age } & Mean \pm SD & $30.53 \pm 12.26$ & & \\
\hline & Range & $13-58$ & & \\
\hline \multirow{2}{*}{ Sex } & Females & $17(56.7 \%)$ & \multirow{2}{*}{0.498} & \multirow{2}{*}{ NS } \\
\hline & Males & $13(43.3 \%)$ & & \\
\hline \multirow{3}{*}{ Side of CB } & Right & $4(13.3 \%)$ & & \\
\hline & Left & $11(36.7 \%)$ & & \\
\hline & Bilateral & $15(50.0 \%)$ & & \\
\hline \multicolumn{3}{|c|}{ Presence of maxillary sinusitis (47) } & p-value & Significance \\
\hline \multirow{2}{*}{ Age } & Mean \pm SD & $37.51 \pm 13.87$ & & \\
\hline & Range & $15-61$ & & \\
\hline \multirow{2}{*}{ Sex } & Females & $26(55.3 \%)$ & \multirow{2}{*}{0.166} & \multirow{2}{*}{ NS } \\
\hline & Males & $21(44.7 \%)$ & & \\
\hline \multirow[b]{2}{*}{ Side of maxillary sinusitis } & Right & $\begin{array}{c}5(10.6 \%) \\
10(213 \%)\end{array}$ & & \\
\hline & $\begin{array}{l}\text { Left } \\
\text { Bilateral }\end{array}$ & $\frac{10(21.3 \%)}{32(68.1 \%)}$ & & \\
\hline
\end{tabular}




\section{Prevalence and severity of Maxillary sinusitis}

From the 75 scans evaluated in this study, 47 (62.7\%) of patients showed evidence of maxillary sinusitis. $21(44.7 \%)$ were males and $26(55.3 \%)$ were females. Again, although females showed higher prevalence of maxillary sinusitis, there was no statistically significant difference between both genders $(\mathrm{p}=0.166)$.

From both genders, 32 (68.1\%) had bilateral, 5 (10.6\%) had right, and 10 (21.3\%) had left maxillary sinusitis. The mean age of patients with maxillary sinusitis was $37.51 \pm 13.87$ years and their age range was from 15 - 61 years (Table 1).

\section{Correlation between NSD \& CB}

In this study, the correlation between NSD and $\mathrm{CB}$ was not statistically significant $(\mathrm{p}=0.559)$. From the 28 patients with NSD, only 10 (35.7\%) showed the presence of $\mathrm{CB}$ and from the 30 patients with $\mathrm{CB}$, $10(33.3 \%)$ showed the presence of NSD and $13.3 \%$ of all patients had a combination of both (Table 2).

Regarding the correlation between the side of NSD and the side of CB, this study revealed that among the 10 patients who showed the presence of both NSD and CB, 4(40\%) patients had bilateral CB, $6(60 \%)$ had CB on the opposite side of the NSD and no patients $(0 \%)$ showed the presence of $\mathrm{CB}$ on the same side of NSD. Here, there was a statistically significant correlation between the presence of NSD and contralateral $\mathrm{CB}(\mathrm{p}=0.032)$ (Table 3).

TABLE (2): Correlation between NSD, CB and MS (maxillary sinusitis)

\begin{tabular}{|c|c|c|c|c|c|}
\hline & & \multicolumn{2}{|c|}{ NSD (28 cases) } & \multirow{2}{*}{ p-value } & \multirow{2}{*}{ Significance } \\
\hline & & Present & Absent & & \\
\hline \multirow{2}{*}{ CB } & Present & $10(35.7 \%)$ & $20(42.6 \%)$ & \multirow{2}{*}{0.559} & \multirow{2}{*}{ NS } \\
\hline & Absent & $18(64.3 \%)$ & $27(57.4 \%)$ & & \\
\hline & & \multicolumn{2}{|c|}{ CB (30 cases) } & \multirow{2}{*}{ p-value } & \multirow{2}{*}{ Significance } \\
\hline & & Present & Absent & & \\
\hline \multirow{2}{*}{ NSD } & Present & $10(33.3 \%)$ & $18(40.0 \%)$ & \multirow{2}{*}{0.559} & \multirow{2}{*}{ NS } \\
\hline & Absent & $20(66.7 \%)$ & $27(60.0 \%)$ & & \\
\hline & & \multicolumn{2}{|c|}{ NSD ( 28 cases) } & \multirow{2}{*}{ p-value } & \multirow{2}{*}{ Significance } \\
\hline & & Present & Absent & & \\
\hline \multirow{2}{*}{ MS } & Present & $19(67.9 \%)$ & $28(59.6 \%)$ & \multirow{2}{*}{0.473} & \multirow{2}{*}{ NS } \\
\hline & Absent & $9(32.1 \%)$ & $19(40.4 \%)$ & & \\
\hline & & \multicolumn{2}{|c|}{ MS (47 cases) } & \multirow{2}{*}{ p-value } & \multirow{2}{*}{ Significance } \\
\hline & & Present & Absent & & \\
\hline \multirow{2}{*}{ NSD } & Present & $19(40.4 \%)$ & $9(32.1 \%)$ & \multirow{2}{*}{0.473} & \multirow{2}{*}{ NS } \\
\hline & Absent & $28(59.6 \%)$ & $19(67.9 \%)$ & & \\
\hline & & \multicolumn{2}{|c|}{ CB (30 cases) } & \multirow{2}{*}{ p-value } & \multirow{2}{*}{ Significance } \\
\hline & & Present & Absent & & \\
\hline \multirow{4}{*}{ MS } & Present & $18(60.0 \%)$ & $29(64.4 \%)$ & \multirow{2}{*}{0.697} & \multirow{2}{*}{ NS } \\
\hline & Absent & $12(40.0 \%)$ & $16(35.6 \%)$ & & \\
\hline & & \multicolumn{2}{|c|}{ MS (47 cases) } & \multirow{2}{*}{ p-value } & \multirow{2}{*}{ Significance } \\
\hline & & Present & Absent & & \\
\hline \multirow{2}{*}{$\mathrm{CB}$} & Present & $18(38.3 \%)$ & $12(42.9 \%)$ & 0697 & NS \\
\hline & Absent & $29(61.7 \%)$ & $16(57.1 \%)$ & & \\
\hline
\end{tabular}




\section{Correlation between NSD \& Maxillary sinusitis}

Additionally, the current study probed the potential correlation between NSD and maxillary sinusitis. Again, there was no statistical significant correlation between them $(\mathrm{p}=0.473)$. From the 28 patients with NSD, 19 (67.9\%) also showed the presence of maxillary sinusitis. On the other hand, from the 47 patients with maxillary sinusitis, 19 (40.4\%) had a deviated septum and $25.3 \%$ of the examined population showed a combination of both (Table 2).

Regarding the relation between the direction of NSD and side of maxillary sinusitis, 5 (26.3 $\%)$ of patients with NSD had ipsilateral maxillary sinusitis, $5(26.3 \%)$ had contralateral sinusitis and 9 $(47.3 \%)$ had bilateral maxillary sinusitis. There was no statistically significant correlation between the side of deviation and the side of sinusitis $(\mathrm{p}=0.512)$ (Table 3).
In this study, the maximum extension of NSD in patients with sinusitis showed a mean of (6.61 \pm 1.44 ) and a range from $4.4-10.03$. There was no statistically significant association between the presence of maxillary sinusitis and maximum extension of NSD ( $\mathrm{p}=0.239)$ (Table 3).

\section{Correlation between CB \& Maxillary sinusitis}

By examining the potential correlation between the presence of $\mathrm{CB}$ and maxillary sinusitis, this study showed that there was no statistical significant correlation $(\mathrm{p}=0.697)$. From the 30 patients with CB, 18 (60.0\%) also had maxillary sinusitis and from the 45 patients without CB, 29 (64.4\%) had maxillary sinusitis (Table 2). In this study the correlation between the presence of $\mathrm{CB}$ and the severity of maxillary sinusitis was also explored. There was a non-statistically significant correlation between the presence of $\mathrm{CB}$ and the severity of maxillary sinusitis ( $\mathrm{p}=0.147$ ) (Table 3).

TABLE (3): Correlation between direction of NSD and side of CB, CB and severity of maxillary sinusitis and between sinusitis and maximum extension of deviation

\begin{tabular}{|c|c|c|c|c|c|}
\hline & \multicolumn{2}{|c|}{ Direction of NSD } & \multirow{2}{*}{ p-value } & \multirow{2}{*}{ Significance } \\
\hline & & Right & Left & & \\
\hline \multirow{3}{*}{ Side of Concha bullosa } & Right & $0(0.0 \%)$ & $1(25.0 \%)$ & \multirow{3}{*}{0.032} & \multirow{3}{*}{$\mathbf{S}$} \\
\hline & Left & $5(83.3 \%)$ & $0(0.0 \%)$ & & \\
\hline & Bilateral & $1(16.7 \%)$ & $3(75.0 \%)$ & & \\
\hline & & \multicolumn{2}{|c|}{ Presence of CB } & \multirow{2}{*}{ p-value } & \multirow{2}{*}{ Significance } \\
\hline & & Absent & Present & & \\
\hline & Mild & $31(62.0 \%)$ & $14(50.0 \%)$ & & \\
\hline \multirow[t]{2}{*}{ Severity of maxillary sinusitis } & Moderate & $12(24.0 \%)$ & $10(35.7 \%)$ & 0.147 & NS \\
\hline & Severe & $7(14.0 \%)$ & $4(14.3 \%)$ & & \\
\hline & & \multicolumn{2}{|c|}{ Presence of Maxillary sinusitis } & \multirow{2}{*}{ p-value } & \multirow{2}{*}{ Significance } \\
\hline & & Absent & Present & & \\
\hline Maximum extension of deviation & $\begin{array}{l}\text { Mean } \pm S D \\
\text { Range }\end{array}$ & $\begin{array}{c}5.82 \pm 1.95 \\
4-10\end{array}$ & $\begin{array}{l}6.61 \pm 1.44 \\
4.4-10.03\end{array}$ & 0.239 & NS \\
\hline & & \multicolumn{2}{|c|}{ Direction of NSD } & \multirow{2}{*}{ p-value } & \multirow{2}{*}{ Significance } \\
\hline & & Right & Left & & \\
\hline Side of maxillary sinusitis & $\begin{array}{l}\text { Right } \\
\text { Left } \\
\text { Bilateral }\end{array}$ & $\begin{array}{l}3 \\
4 \\
4\end{array}$ & $\begin{array}{l}1 \\
2 \\
5\end{array}$ & 0.512 & NS \\
\hline
\end{tabular}




\section{DISCUSSION}

The good image quality provided by $\mathrm{CBCT}$, its relatively low radiation dose and its simple and user-friendly software made it the method of choice in many maxillofacial applications. Additionally, CBCT provides excellent contrast between boneair and mucosa, allowing the ability to study the anatomy and pathology of both the nasal cavity and paranasal sinuses. In particular, coronal CBCT images significantly improved the visualization of the nasal cavity and paranasal sinuses ${ }^{\mathbf{1 0}}$. Therefore, deviation of the nasal septum, presence of concha bullosa, sinus-mucosal thickening, as well as sinus effusion can be perfectly recognized on CBCT images. Soon, CBCT will become the gold standard in routine sinus examination ${ }^{19}$.

It is well known that NSD and CB are common anatomical variations of the nasal cavity and have been described in different studies ${ }^{\mathbf{1 0}, 22}$. The proper alignment of the nasal septum allows the operation of the two sides of the nose which in turn provides both conditioning of the air and respiratory defense ${ }^{13}$.NSD may therefore interfere with nasal physiology as it frequently causes narrowing of the middle nasal meatus which in turn causes nasal obstruction and secondary nasal and sinus infections. These infections are most frequently observed in the maxillary sinus. According to the severity of nasal septum deviation, partial or complete blockage of the respiratory passage may occur ${ }^{23}$.

In this study, the prevalence of NSD, CB and maxillary sinusitis and their correlation was explored. NSD was reported in $37.3 \%$ (28 patients) of the sample size. This came very close to the results obtained by Bahemmat and Hadian ${ }^{11}$ who reported that the prevalence of septal deviation was $37 \%$. In the current study, although the majority $(64.3 \%)$ of the subjects with NSD were females, there was no statistically significant difference between both genders regarding the presence of NSD $(\mathrm{p}=0.685)$. Several studies on the other hand reported that NSD is more commonly seen in males ${ }^{12,24}$. They explained that by the fact that NSD occurs frequently due to trauma which is more commonly seen in males. In this study, however, most CBCT scans used were initially taken for implant and prosthetic treatment which are mainly used for aesthetic purposes, more commonly needed by females, and hence the greater percentage of NSD among females.

In this study, right-sided NSD was seen in 16 $(57.1 \%)$ of the patients, while left-sided NSD was observed in 12 (42.9\%) of the patients. These results came also close to those of Stallman et al. ${ }^{17}$ who reported that septal deviation was to the right in $51 \%$ of their cases and to the left in $49 \%$. As reported by numerous studies, there was no significant predilection to either the right or left side ${ }^{12,24}$. In the current study, the mean of the maximum extension of NSD from the midline was $6.36 \pm 1.63$ $\mathrm{mm}$ and the range was from $4-10.03 \mathrm{~mm}$. Gencer et al ${ }^{9}$,however measured the angle of deviation, and reported that the NSD angle was between $\left(5^{\circ}\right.$ $27.2^{\circ}$ ). It was therefore difficult to compare the results of both studies.

The nasal CB represents the most common anatomical variation in the osteomeatal complex. It is usually seen in the middle nasal conchae, being rare in the superior nasal conchae and even rarer in the inferior nasal conchae ${ }^{12}$. In the literature, the incidence of middle $\mathrm{CB}$ was reported in the range from $13-72.2 \%{ }^{1}$. In this study, the incidence of $\mathrm{CB}$ was $40 \%$ of the examined population. The differences between the studies are probably related to the different ethnic background of the study population.

Among the 30 patients with $\mathrm{CB}, 4$ (13.3\%) were located in the right side, $11(36.7 \%)$ were located in the left side and 15 (50.0\%) were present bilaterally. Several studies also reported that the incidence of bilateral CB ranges from $45.0 \%$ to $61.5 \%{ }^{12}, 25$. Again, these small differences are probably related to the intrinsic variations of the examined study population. 
In this study, although females showed higher prevalence of CB (56.7\%), there was no statistically significant difference between both genders $(\mathrm{p}=0.498)$. These results came in agreement with several other studies as Bahemmat and Hadian ${ }^{11}$, who also reported that $\mathrm{CB}$ is more common among females.

A number of studies examined the prevalence of maxillary sinusitis in different study populations. Bolger et al. ${ }^{26}$ reported that maxillary sinusitis occurred in $83 \%$ of their patients. Bahemmat and Hadian ${ }^{11}$ on the other hand reported a lower prevalence of maxillary sinusitis (33.6\%).This study showed that $62.7 \%$ of the studied population had maxillary sinusitis. Again, these variations are mainly related to the differences between the study populations. Additionally, it should be mentioned that most CBCT scans used in this study were acquired for dental purposes and may show a lower incidence of maxillary sinusitis than other study populations in which patients were referred for CT examination for sinonasal symptoms. Another possible cause of these variations may be related to seasonal bias, as some studies' population were collected during a season in which patients are more vulnerable to allergies. It should be therefore mentioned that the scans used in the current study were collected over an 18 months period (involving different seasons) and are therefore more realistic and representative for the actual situation.

Furthermore, similar to Subramanian et al ${ }^{27}$ who reported a higher prevalence of maxillary sinusitis among females, this study also showed a higher prevalence of maxillary sinusitis among females but again with no significant difference between both genders $(\mathrm{p}=0.166)$. However, Smith et al ${ }^{10}$ reported a statistically significant higher incidence of maxillary sinusitis in males. This difference may be attributed to the mucosal secretion dissimilarities between both genders.

For the correlation between NSD and CB, the results of this study came in agreement with Lopes et al ${ }^{12}$ regarding the fact that NSD did not increase the incidence of formation of CB. On the contrary,
Bahemmat and Hadian ${ }^{11}$ found a significant correlation between NSD and CB. However, regarding the correlation between the side of $\mathrm{CB}$ and the direction of NSD, this study showed that there is a statistically significant correlation between the presence of NSD and the presence of contralateral CB ( $p=0.032)$. Similarly, Uygur et al ${ }^{25}$ and Keles et al ${ }^{1}$ found a strong correlation between the presence of a deviated septum and contralateral CB. As no patients in this study had deviated nasal septum and ipsilateral $\mathrm{CB}$, it could be concluded that NSD may be a factor that prevents the formation of $\mathrm{CB}$ on the same side. Although, the exact mechanism of $\mathrm{CB}$ formation is still not completely understood, airflow is considerably reduced in the nasal cavity on the side of the convexity of nasal septum and pneumatization of the middle concha is increased on the contralateral side ${ }^{1}$. This theory might explain the correlation between NSD and the presence of contralateral middle $\mathrm{CB}$.

For the correlation between NSD and maxillary sinusitis, this study showed that there is no statistical significant correlation between them $(p=0.473)$. Likewise, some other studies rejected such a correlation ${ }^{24,28}$. However, other studies like that of Bahemmat and Hadian ${ }^{11}$ showed that there is a significant correlation between the presence of deviation and sinusitis. Furthermore, Gencer et al ${ }^{9}$ reported that severe deviation may be a contributing factor for sinusitis. Hatipoglu et al. ${ }^{29}$ also found correlation between the degree of septal deviation and the presence of maxillary sinusitis. Contrarily, this study showed no statistically significant association between the presence of maxillary sinusitis and maximum extension of NSD $(p=0.473)$ and between the presence of NSD and severity of maxillary sinusitis $(\mathrm{p}=0.544)$. Similarly, a metaanalysis conducted by Collet et al. ${ }^{30}$ also failed to find a definite relationship between the degree of NSD and presence of maxillary sinusitis. As the patients in this study were selected randomly from the oral and maxillofacial radiology department with no primary sinonasal complaint, we therefore believe that the results of this study are more representative to the whole community and therefore came in 
agreement with the aforementioned meta-analysis. However, it seems that the correlation between NSD and maxillary sinusitis will remain a matter of debate.

Furthermore, in this study there was no association between the direction of the deviated septum and the side of sinus inflammation. These results came in agreement with Stallman et al ${ }^{17}$ who also found no relationship. As we mentioned before, NSD may cause some nasal obstruction and secondary sinus inflammation regardless of the side.

For the correlation between $\mathrm{CB}$ and maxillary sinusitis, some researchers believe that $\mathrm{CB}$ causes sinus obstruction and therefore increases the incidence of maxillary sinusitis ${ }^{27}$. However, others argue that there is no correlation ${ }^{\mathbf{1 7}, \mathbf{2 6}}$. Stallman et al. ${ }^{17}$ reported that $72 \%$ of patients with $\mathrm{CB}$ had sinus disease and that $78 \%$ of patient without $\mathrm{CB}$ had sinus disease. Therefore, they concluded that there was no correlation between the presence of $\mathrm{CB}$ and the presence of sinus disease. Similarly, in the current study about $60 \%$ of patients with CB also had maxillary sinusitis and $64.4 \%$ of patients had sinusitis without $\mathrm{CB}$. As the case with the aforementioned study, there was no statistical significant correlation between the presence of $\mathrm{CB}$ and maxillary sinusitis. A possible explanation for this discrepancy is that those studies which found a correlation, mostly included patients with preexisting sinonasal symptoms. Additionally, this study showed no correlation between the presence of $\mathrm{CB}$ and the severity of maxillary sinusitis. Most of the patients with or without $\mathrm{CB}$ had mild maxillary sinusitis, while the least number of both groups had severe sinusitis. Up to our knowledge, there are no other studies that investigated such a relationship.

\section{CONCLUSIONS}

In this study, maxillary sinusitis was more common than both NSD and CB. There was no difference between genders in all examined parameters. There was also no correlation between NSD and CB, NSD and maxillary sinusitis, and $\mathrm{CB}$ and maxillary sinusitis. However, there was an association between the presence of NSD and contralateral CB. Additionally, no correlation was found between presence of maxillary sinusitis and maximum extension of NSD, presence of $\mathrm{CB}$ and severity of maxillary sinusitis, nor between the direction of NSD and side of sinusitis. From the previous results it could be concluded that neither NSD, nor the presence of $\mathrm{CB}$ increases the risk factor for development of sinusitis.

\section{REFERENCES}

1- Keles Bahar, Ozturk Kayhan, Unald Deniz. Is there any relationship between nasal septal deviation and concha bullosa. Eur J Gen Med 2010; 7(4):359-364.

2- Orhan I, Aydin S, Ormeci T, Yilmaz F. A radiological analysis of inferior turbinate in patients with deviated nasal septum by using computed tomography. Am J Rhinol Allergy 2014; 28: 68-72.

3- Egeli E, Demirci L, Yazycy B, Harputluoglu U. Evaluation of the inferior turbinate in patients with deviated nasal septum by using computed tomography. Laryngoscope 2004; 114: 113-17.

4- R. K. Mundra, Yamini Gupta, Richi Sinha, Alaknanda Gupta.CT scan study of influence of septal angle deviation on lateral nasal wall in patients of chronicr. Indian J Otolaryngol Head Neck Surg 2014; 66(2):187-190.

5- Roblin DG, Eccles R. What, if any, is the value of septal surgery? Clin Otolaryngol.2002; 27:77-80.

6- Van Egmond M., Rovers M., Hendriks C, van Heerbeek N. Effectiveness of septoplasty versus nonsurgical management for nasal obstruction due to a deviated nasal septum in adults: study protocol for a randomized controlled trial. Trials 2015;16:500.

7- Daghistani KJ. Nasal septal deviation in Saudi patients: a hospital based study. J KAU Med Sci 2002; 10:39-46.

8- Wang J, Dou X, Liu D, Song P, Qian X, Wang S, et al. Assessment of the effect of deviated nasal septum on the structure of nasal cavity. Eur Arch Otorhinolaryngol. 2016; 273(6):1477-80.

9- Zeliha Kapusuz Gencer, Mahmut Ozkiris, Aylin Okur, Seyhan Karacavu,Levent Saydam. The effect of nasal septal deviation on maxillary sinus volumes and development of maxillary sinusitis. Eur Arch Otorhinolaryngol 2013; 270:3069-3073. 
10- Kyle D. Smith, Paul C. Edwards, Tarnjit S. Saini, Neil S. Norton. The prevalence of Concha Bullosa and Nasal Septal Deviation and their relationship to Maxillary Sinusitis by Volumetric Tomography. International Journal of Dentistry 2010; Article ID 404982.

11- Nika Bahemmat and Hoora Hadian. The frequency of nasal septal deviation and concha bullosa and their relationship with maxillary sinusitis based on $\mathrm{CBCT}$ finding. International Journal of Medical Research \& Health Sciences 2016; 5(11):152-156.

12- Sérgio Lopes, Mari de Moraes, Luiz Coutinho et al. ConeBeam Computed Tomography analysis of prevalence of Nasal Septum Deviation and its relationship with the presence of Middle Concha Bullosa. Braz Dent Sci 2015; 18(2): 38-43.

13- Ismail Serifoglu, Ibrahim Ilker Oz, Murat Damar, et al. Relationship between the degree and direction of nasal septum deviation and nasal bone morphology. Head \& Face Medicine; 2017,13(3).

14- Fatma Çaglayan, Ümmühan Tozoglu. Incidental findings in the maxillofacial region detected by cone beam CT. Diagn Interv Radiol 2012; 18:159-163.

15- S. Subramanian, G. R. L. Rampal, E. F. M. Wong, S. Mastura, and A. Razi. Concha bullosa in chronic sinusitis. Medical Journal of Malaysia 2005;60(5), 535-539.

16- J. S. Lee, J. Ko, H. D. Kang, and H. S. Lee. Massive concha bullosa with secondary maxillary sinusitis. Clinical and Experimental Otorhinolaryngology 2008; 1(4), 221-223.

17- J. S. Stallman, J. N. Lobo, and P. M. Som. The incidence of concha bullosa and its relationship to nasal septal deviation and paranasal sinus disease. American Journal of Neuroradiology 2004; 25( 9), 1613-1618.

18- S. A. R. Nouraei, A. R. Elisay, A. DiMarco et al. Variations in paranasal sinus anatomy: implications for the pathophysiology of chronic rhinosinusitis and safety of endoscopic sinus surgery. Journal Otolaryngology - Head \& Neck Surgery 2009;38(1), 32-37.

19- C. Hodeza, C. Griffaton-Taillandier, I. Bensimon. Cone-beam imaging: Applications in ENT. European Annals of Otorhinolaryngology, Head and Neck diseases 2011;128, 65-78.

20- Gökhan Göçme, Mehmet Ouz Borahan, Sertac Aktop, Asim Dumlu, Filiz Namdar Pekiner and Kamil Göker.
Effect of Septal Deviation, Concha Bullosa and Haller's Cell on Maxillary Sinus's inferior pneumatization; a Retrospective Study. The Open Dentistry Journal, 2015; 9 , (Suppl 2: M5) 282-286.

21- Rak KM, Newell JD 2nd, Yakes WF, Damiano MA, Luethke JM. Paranasal sinuses on MR images of the brain: significanc of mucosal thickening. AJR Am J Roentgenol 1991; 156:381-384.

22- Sevinc O, Barut C, Kacar D, Is M. Evaluation of the lateral

23- wall of the nasal cavity in relation to septal deviation. Int $\mathbf{J}$ Morpho. 2013; 31: 438-443.

24- Kapusuz Gencer Z, Ozkiris M, Okur A, Karacavus S, Saydam L. The effect of nasal septal deviation on maxillary sinus volumes and development of maxillary sinusitis. Eur Arch Otorhinolaryngol. 2013; 270(12):3069-73.

25- Lin JK, Wheatley FC, Handwerker J, Harris NJ, Wong BJ. Analyzing nasal septal deviations to develop a new classification system: a computed tomography study using MATLAB and OsiriX. JAMA Facial Plast Surg. 2014 May-Jun;16(3):183-7.

26- Uygur K, Tüz M, Dogru H. The correlation between septal deviation and concha bullosa. Otolaryngol Head Neck Surg. 2003;129 (1):33-6.

27- Bolger WE, Parsons DS, Butzin CA. Paranasal sinus bony anatomic variations and mucosal abnormalities: CT analysis for endoscopic sinus surgery. The Laryngoscope. 1991;101(1):56-64.

28- Subramanian S, Lekhraj RG, Wong E, Mastura S, Razi A. Concha bullosa in chronic sinusitis. The Medical journal of Malaysia 2005;60(5):535-9.

29- Hamdan A, Bizri A, Jaber M, Hammoud D, Baino T, Fuleihan N. Nasoseptal variation in relation to sinusitis. A computerized tomographic evaluation. The Lebanese medical journal. 2000;49 (1):2-5.

30- Hatipoglu HG, Cetin MA, Yuksel E (2008) Nasal septal deviation and concha bullosa coexistence: CT evaluation. B-ENT 4:227-232.

31- S. Collet, B. Bertrand, S. Cornu, P. Eloy, and P. Rombaux, "Is septal deviation a risk factor for chronic sinusitis? Review of literature," Acta Oto-Rhino-Laryngologica Belgica 2001; 55(4), 299-304. 